\title{
Famílias em Vulnerabilidade Social: Rastreamento de Termos Utilizados por Terapeutas de Família
}

\author{
Laíssa Eschiletti Prati ${ }^{1}$ \\ Maria Clara P. de Paula Couto \\ Sílvia Helena Koller \\ Universidade Federal do Rio Grande do Sul
}

\begin{abstract}
RESUMO - Este estudo investigou se os termos "famílias em situação de risco", "famílias pobres", "famílias de camadas populares" e "famílias de baixa renda" podem referir-se ao conceito de "famílias em vulnerabilidade social", quando utilizados por terapeutas de família. Foi realizada uma pesquisa documental em anais de seis congressos nacionais e de um congresso internacional de terapia familiar que aconteceu no Brasil. Efetuou-se uma análise quantitativa que visou a enumeração de quantas vezes esses termos foram utilizados e uma qualitativa que objetivou rastrear a definição apresentada para os mesmos. Entre 1994 e 2006 houve um aumento de nove para 21 trabalhos utilizando os termos investigados. O material bibliográfico revelou que os diversos termos são complementares e se referem, em geral, a famílias em vulnerabilidade social.
\end{abstract}

Palavras-chave: terapia familiar; nível sócio-econômico; vulnerabilidade; resiliência.

\section{Families in Social Vulnerability: Survey on Terms Used by Family Therapists}

\begin{abstract}
This study investigated if the terms "families at risk", "poor families", "families of popular class" and "low income families" could be related to the concept of "families in social vulnerability", when used by family therapists. It was performed a documental survey on the proceedings of six Brazilian scientific conferences and of one international conference about family therapy that occurred in Brazil. A quantitative analysis was conducted in order to verify how often these terms were used. A qualitative analysis was also performed in order to identify their definition. From 1994 to 2006, there was an increase from nine to 21 works using the investigated terms. The bibliographic material showed that these different terms are complementary and generally related to families in social vulnerability.
\end{abstract}

Keywords: family therapy; socioeconomic status; vulnerability; resilience.

\section{Percurso da Terapia Familiar no Brasil por meio da Leitura de Anais de Congressos (de 1994 a 2006)}

A terapia familiar é relativamente nova no cenário brasileiro. Segundo Aun (2005), os terapeutas familiares constituíram-se enquanto grupo no final da década de 70. Importando modelos e teorias de outras culturas, os profissionais precisaram adaptar-se às especificidades de uma realidade que exigia a construção de novas intervenções. Visando ao desenvolvimento saudável das mais diversas caracterizações familiares, os terapeutas precisaram lidar com incertezas e criar estratégias de sobrevivência.

Uma das estratégias utilizadas pelos terapeutas familiares para construir uma base sólida e coerente foi a realização de congressos nacionais. Ao mesmo tempo em que esses encontros permitem a visibilização das atividades que são realizadas por esse grupo de profissionais, também possibilita uma maior integração de referenciais e uma apropriação de termos de forma mais coerente. Por meio da leitura atenta das publicações de anais dos congressos nacionais é possível perceber as direções gerais adotadas na história da terapia

1 Endereço para correspondência: Av. Cavalhada 4760, B1. A12, Apto. 346, Cavalhada. Porto Alegre, RS. CEP 91740-000. Fone: (51) 3377.2814 ou 9335.0354. Fax: (51) 3249.4255.E-mail: laissa.prati@ terra.com.br ou laissa.prati@gmail.com. familiar brasileira. Aun (2005) aponta que, ao observar os temas centrais de cada congresso, fica evidente uma tendência de ampliação dos conceitos de família e de terapia.

O primeiro congresso aconteceu em São Paulo, no ano de 1994, com o tema norteador "Família: lugar seguro para crescer?". Foi o primeiro momento no qual os terapeutas de família trocaram idéias em âmbito nacional. Nessa ocasião, foi fundada a Associação Brasileira de Terapia Familiar (ABRATEF). No segundo congresso, realizado em Gramado em 1996, não foi definido um tema. Gonçalves (1996), ao sugerir alguns motivos para a ausência de tema no congresso, afirma que os terapeutas de família lidam com uma diversidade de relações do indivíduo, sendo impossível encontrar uma única teoria que dê conta de todas as situações que surgem na prática clínica. Segundo esse autor, é necessária a integração de modelos e a busca por uma atitude de abertura e capacidade de questionamento.

O terceiro congresso ocorreu no Rio de Janeiro, em 1998. $\mathrm{O}$ tema central foi "O indivíduo, a família e as redes sociais na virada do século". Nesse mesmo ano, Rapizo (1998) apresenta em seu livro "Terapia sistêmica de família - da instrução à construção", a inserção de um modelo construtivista de terapia familiar. Afirma que tal postura epistemológica não apresenta uma forma de fazer terapia, mas abre a possibilidade para novos modelos clínicos e teóricos, criando 
um contexto criativo para a terapia familiar brasileira. Esse espaço de construção pode ser percebido na evolução dos congressos que se seguiram.

O quarto congresso ocorreu em Brasília, em 2000, com o tema "A família em tempos de transição: justiça social, ética e cidadania". Aun (2005) enfatiza que nesse congresso foram enfocadas questões referentes à responsabilidade social do profissional que trabalha com famílias. Nesse congresso, apareceu de forma mais evidente uma abertura a aspectos relativos às políticas públicas e ao questionamento (e consequente ampliação) do papel do terapeuta familiar para a sociedade brasileira.

Um ano após os terapeutas familiares refletirem sobre seu papel social, uma atividade de visibilidade internacional aconteceu no Brasil. Em 2001, o XIII Congresso Internacional de Terapia Familiar da International Family Therapy Association (IFTA) foi sediado em Porto Alegre. O tema central foi "Desafios à família no século 21 - teoria, prevenção e terapia". Foi possível a acolhida a uma diversidade de temas que refletiam o dia-a-dia das famílias brasileiras e do mundo. A discussão entre países evidenciou que o Brasil já tinha conquistado uma forma particular de lidar com famílias e com sua influência na sociedade. Salientou-se a criatividade e a esperança como aspectos bastante valorizados nos atendimentos de família realizados no Brasil.

$\mathrm{O}$ quinto congresso aconteceu em Salvador, em 2002. O tema foi "Família com afeto". Houve um resgate da família que busca atendimento psicológico, porém uma atenção especial ao papel do terapeuta familiar também foi observada. Foi considerado que o terapeuta possui um papel chave na evolução da terapia e precisa ser valorizado.

O sexto congresso, ocorrido em Florianópolis, em 2004, trabalhou com o tema "O que tu fazes por aí? Diversidades e abordagens da família brasileira". Nesse momento da terapia familiar brasileira foi importante discutir e apresentar os inúmeros espaços que estavam sendo trabalhados a partir de uma visão sistêmica. Novamente, a integração dos profissionais salientou a infinidade de locais que demandam a presença atenta do terapeuta familiar. Foram bastante enfatizados os trabalhos em centros de saúde e as atuações junto às camadas menos favorecidas da sociedade. O terapeuta familiar não atua somente no consultório, está na sociedade.

O sétimo congresso aconteceu em São Paulo, no ano de 2006. O tema em estudo foi "Tecendo redes e construindo pontes entre teorias, práticas e contextos". Esse congresso apresentou duas ênfases: um desejo por parte dos terapeutas em tentar identificar os caminhos trilhados desde 1994 e uma intenção em apresentar as bases teóricas que estavam sendo utilizadas na prática clínica.

A análise dos anais dos congressos nacionais e do congresso internacional que ocorreu no Brasil evidencia uma imensa diversidade terminológica e conceitual. Termos são utilizados como sinônimos, sem haver rigor quanto ao que deveriam significar. Existem termos que são "modismos", utilizados por diversos profissionais com entendimentos diferenciados. Outros termos emergem do senso comum e, portanto, não têm sua conceituação explicitada. Há uma sensação de unidade que diminui a necessidade de tal rigor teórico. Hoje, entretanto, há uma preocupação com a construção de um campo conceitual coerente e sistema- tizado para o embasamento das atividades executadas por terapeutas familiares.

Diversos aspectos relevantes à prática clínica dos terapeutas familiares articulam-se aos termos famílias em situação de risco, famílias pobres, famílias de camadas populares e famílias de baixa renda. A relevância desses termos é confirmada por sua utilização em diversos trabalhos científicos apresentados em congressos de terapia familiar. Portanto, este estudo pretende verificar como esses termos se relacionam ao conceito de 'famílias em vulnerabilidade social' por meio da análise documental dos anais dos congressos dessa área realizados no Brasil.

\section{Conceituação de Famílias em Vulnerabilidade Social}

O campo de estudo sobre famílias em vulnerabilidade social tem sido bastante explorado pelas investigações sobre terapia de família. No entanto, em decorrência da multidisciplinaridade característica desse campo de atuação e pesquisa, evidencia-se certa imprecisão conceitual na utilização do termo "famílias em vulnerabilidade social". O surgimento de ambivalências e contradições torna-se inevitável quando profissionais com apoios teóricos diferentes passam a trabalhar com um público com uma problemática particular e cada vez mais frequente na realidade brasileira.

Tomando como ponto de partida a conceitualização de família como um sistema interacional em desenvolvimento, a vulnerabilidade social pode ser expressa no adoecimento de um ou vários membros, em situações recorrentes de uso de drogas, violência doméstica e outras condições que impeçam ou detenham o desenvolvimento saudável desse grupo. Vulnerabilidade social é uma denominação utilizada para caracterizar famílias expostas a fatores de risco, sejam de natureza pessoal, social ou ambiental, que coadjuvam ou incrementam a probabilidade de seus membros virem a padecer de perturbações psicológicas. Tais riscos estão, em geral, associados a eventos de vida negativos, que potencializam e predispõem a resultados e processos disfuncionais de ordem física, social e/ou emocional.

Assim, pode ser considerado como risco todo evento que se configure como obstáculo em nível individual ou contextual e que potencialize a vulnerabilidade inerente (Pesce, Assis, Santos \& Carvalhaes, 2004). Frente aos riscos, o sistema familiar pode responder de forma ineficaz, não tendo condições de reagir funcionalmente e de estabelecer uma base saudável para seu desenvolvimento. Fontes de recursos estruturais que compõem a rede de apoio social e afetivo dessas famílias não conseguem mais ser acionadas de forma funcional e responsiva. A coesão ecológica interna à família está abalada e não consegue desenvolver saídas que promovam resiliência e superação.

O termo vulnerabilidade surgiu no campo da Terapia Familiar em uma perspectiva individualista (Walsh, 1996). Ao longo dos anos, tal perspectiva foi se ampliando e levando em conta o sistema em estudo - partindo de uma pessoa para uma família e/ou para uma comunidade. Não só o indivíduo, mas todo o sistema passou a ser percebido como podendo apresentar-se fragilizado ao ter que lidar com situações de 
risco. Quando o sistema se percebe como vulnerável, surgem sentimentos de impotência, de falta de recursos e de fragilização, que o conduzem à paralisação e/ou à doença. Um sistema exposto a situações indesejáveis, que percebe que suas tentativas de resolver impasses podem não ser suficientes, apresenta vulnerabilidade. Quanto mais vulnerável estiver, maior a predisposição para apresentar problemas no desenvolvimento (Cecconello, 2003; Cowan, Cowan \& Schulz, 1996; Masten \& Garmezy, 1985).

O termo família em vulnerabilidade social surge, portanto, dessa forma de compreender o desenvolvimento humano individualizado. Famílias vulneráveis são aquelas nas quais os indivíduos que a compõem apresentam déficits em seus recursos pessoais. Assim, diversos termos são utilizados para descrever esse público como, por exemplo, famílias problema e famílias disfuncionais.

Aos poucos, surgiram defensores de uma abordagem baseada nos aspectos positivos do sistema que substituíram a expressão famílias multiproblemáticas por famílias em alto risco ou multinecessitadas (Rutter, 1999; Walsh, 1998). Tal ênfase em aspectos positivos foi derivada de uma preocupação em ampliar o campo da Psicologia, modificando o foco dos estudos. Objetivava-se não restringir as investigações a pontos negativos, buscando reparar o que estava errado ou ruim, mas reforçando e reconstruindo qualidades positivas (Seligman, 2002). Esse foco mais positivo permitiu o acesso ao estudo da resiliência e deu visibilidade aos fatores de proteção. Rutter (1987) argumentou que os fatores de proteção só atuam ou têm efeito na presença de um fator de risco. Ou seja, populações que não estão expostas a potenciais fatores de risco só virão a acionar os fatores protetores estando na presença de pelo menos um fator de risco.

Com esse movimento, famílias vulneráveis começaram a ser compreendidas como sistemas carentes de suporte social, repletas de desafios e necessidades não correspondidas. As situações de crise surgem na interação da família com a comunidade e com a sociedade em geral. Todos esses fatores devem ser considerados no rastreamento de recursos (fatores de proteção) que podem ser acionados pela família em desenvolvimento.

A resiliência refere-se ao processo que atua na presença do risco e através do qual são produzidos resultados tão bons ou ainda melhores do que os obtidos na ausência de adversidade. Assim, a resiliência é revelada pela boa adaptação dos indivíduos ao seu contexto e à sua vida, mesmo diante de situações de risco (Cowan \& cols., 1996). Dessa forma, a resiliência pode ser compreendida como o resultado dos processos de confronto entre o indivíduo e as circunstâncias específicas em que se encontra.

Embora no trabalho com famílias em vulnerabilidade social o discurso mais marcante ainda se refira aos fatores de risco, há uma tentativa de quebra do discurso de incapacidade de lidar com as dificuldades da vida. Pode-se dizer, então, que os terapeutas buscam fatores de proteção "esquecidos" pelo sistema e a promoção da resiliência em oposição à vulnerabilidade. Ao investigar os fatores de proteção, os terapeutas buscam características que diminuem a probabilidade de um resultado negativo ou indesejado acontecer na presença de um fator de risco, reduzindo a sua incidência e a sua severidade (Cowan \& cols., 1996).
A leitura atenta dos anais dos congressos evidenciou da recorrência do uso de termos como famílias em situação de risco, famílias pobres, famílias de camadas populares e de famílias de baixa renda. O presente trabalho objetivou investigar se esses termos, frequentemente utilizados por terapeutas de família, podem se referir ao conceito de famílias em vulnerabilidade social, conforme descrito nesta sessão.

\section{Método}

O método adotado neste estudo foi a análise bibliográfica. Assim, foi feita a leitura dos anais dos trabalhos apresentados em seis congressos brasileiros de terapia familiar (1994, 1996, 1998, 2002, 2004 e 2006). Os resumos foram acessados através de livros impressos, CDs de divulgação ou material disponível no site da ABRATEF. O material do quarto congresso (realizado em 2000) não foi encontrado em nenhuma dessas fontes. Sendo assim, como uma forma de substituição, optou-se por incluir os anais do XIII Congresso Internacional da IFTA, realizado em 2001, em Porto Alegre, RS.

Para analisar os documentos, foram escolhidos os seguintes termos: famílias em situação de risco, famílias pobres, famílias de camadas populares e famílias de baixa renda. Esses termos foram rastreados e contados a fim de se verificar a frequência com que foram utilizados ao longo dos anos. Em seguida, foi feita uma nova leitura com o objetivo de identificar a forma como esses termos eram definidos pelos autores dos trabalhos publicados nos anais. Finalmente, integraram-se os conceitos encontrados nos anais com vistas a avaliar de que forma os mesmos se relacionavam com o conceito teórico de famílias em vulnerabilidade social.

\section{Resultados e Discussão}

A análise bibliográfica do material pesquisado revelou um aumento expressivo do número de vezes que os diversos termos investigados são utilizados pelos terapeutas de família ao longo dos anos.

Na Figura 1 aparece um aumento no número de trabalhos que tratam de "famílias em situação de risco", "famílias pobres", "famílias de camadas populares" e "famílias de baixa renda" (de nove trabalhos apresentados no primeiro congresso para 21 no último). O congresso de 2004 é o que teve um maior número de trabalhos que utilizaram esses termos (39 trabalhos). O número elevado de trabalhos nesse ano pode indicar o quanto a atuação junto a esse público estava exigindo dos profissionais. Acredita-se que congressos nacionais são espaços de excelência para a reflexão e trocas sobre as situações que mais intrigam os profissionais. $\mathrm{O}$ número acentuado de trabalhos, portanto, pode indicar a necessidade de construir uma forma de trabalho mais embasada teoricamente ou mais difundida entre os profissionais.

A análise das definições dos termos indicou que os autores, quando os definem, apresentam diversos aspectos que se referem a famílias em vulnerabilidade social. Por exemplo, Dos Santos (2004) define as famílias de baixa renda como sendo aquelas que vivem em situações desfavoráveis em termos de acesso aos bens sociais, saúde, la- 

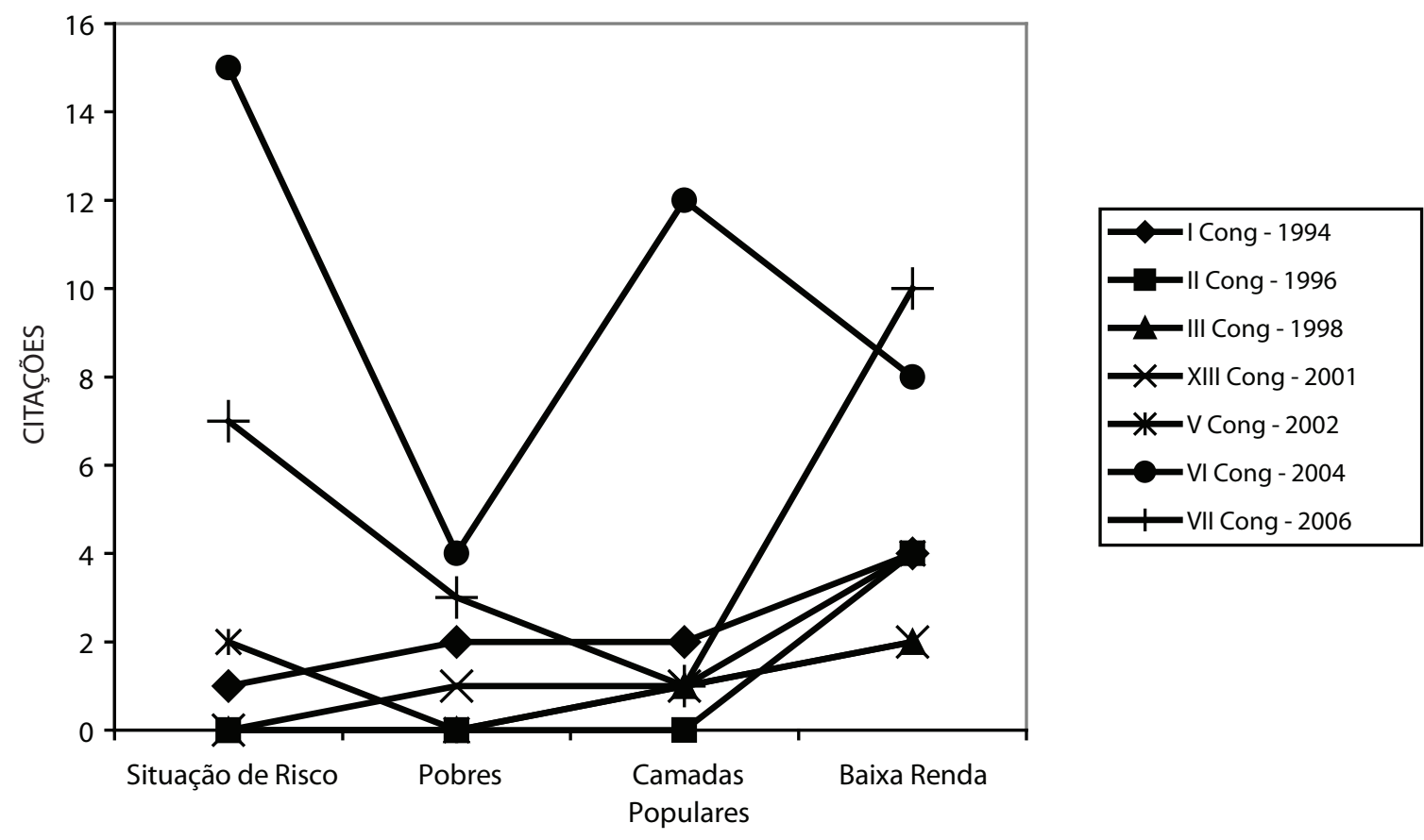

CONCEITOS

Figura 1. Família em vulnerabilidade social - sinônimos

zer, educação, moradia etc. As características psicológicas das famílias em situação de risco, conforme Kuperman, em trabalho apresentado no congresso de 2004, são confusão de identidade social e cultural e baixa auto-estima. Alguns resumos apresentam aspectos sobre as relações familiares, salientando que as relações conjugais caracterizam-se por forte instabilidade; o pai aparece como uma figura afetivamente ausente, mesmo estando fisicamente presente. Apesar de altas incidências de mulheres sem parceiros, com muitos filhos de pais variados, a estrutura hierárquica ainda é estabelecida de forma patriarcal, de acordo com alguns trabalhos do congresso de 1994 (e.g., Martinez, 1994; Sarti, 1994).

Conforme apontado em alguns dos resumos apresentados nos congressos investigados, as dificuldades financeiras são um fator de risco para a manutenção dos relacionamentos familiares que, nessas famílias de baixa renda, tendem a se deteriorar em função da constante luta pela sobrevivência (e.g., Bizzo, 1994). A ausência de condições básicas de sobrevivência, o convívio com uma configuração familiar empobrecida e vida familiar com laços afetivos frágeis são componentes que definem a família em situação de risco. Crises simultâneas e/ou sucessivas são apresentadas como desencadeadoras de desamparo e isolamento dos indivíduos dessas famílias (e.g., Prado, 2001; Timoteo \& Falcão, 2004; Vilela, Gusmão \& Cavana, 2004). A importância da rede de apoio social é destacada por Silveira (1998), que afirma que o apoio para a sobrevivência das famílias é viabilizado pela formação de laços de parentesco e de vizinhança. As famílias operam arranjos compensatórios da ausência masculina no núcleo familiar através de uma rede mais ampla, das relações de vizinhança e de redes que ultrapassam o núcleo doméstico (Sarti, 1994).
As famílias de camadas populares são apresentadas como um eterno desafio às equipes de saúde mental no trabalho de Felzenswalb (1994), que afirma que elas acumulam contatos com diversos sistemas sociais. Assim, cada sistema público fica assoberbado pela mistura de problemas concretos ligados à sobrevivência na vida cotidiana e à desorganização emocional e psicológica.

A família de baixa renda foi caracterizada por Martinez (1994) como altamente carente e dependente do sistema social. Tejadas (2002) contextualiza a situação de dependência dessas famílias apontando para uma mudança de visão. Assim, se os programas públicos enfocavam as famílias pobres como desprovidas de autonomia e de capacidade de gerenciar sua própria vida, os programas mais recentes apontam para a cidadania como um objetivo e a família como um sujeito de direitos, com capacidade de decisão.

Esses resultados permitiram constatar uma tendência dos terapeutas de família em abordar os termos famílias em situação de risco, pobres, de camadas populares e de baixa renda como fazendo referência a famílias em vulnerabilidade social. Esses termos estão relacionados a um mesmo tipo de família, geralmente associada a condições de pobreza e risco social. Sendo assim, a partir desses resultados, pode-se dizer que todos esses termos se referem ao conceito de famílias em vulnerabilidade social.

\section{Considerações Finais}

A partir da análise das variações da utilização dos termos ao longo dos anos, dados relativos à atualização teórica puderam ser inferidos. Constatou-se que os termos famílias em situação de risco, famílias pobres, famílias de 
camadas populares e familias de baixa renda foram utilizados como referência a uma mesma população (famílias em vulnerabilidade social), com pouca precisão conceitual. A pobreza pode surgir como um fator de risco para determinadas famílias e para outras não, dependendo da existência de fatores que moderem seus efeitos (fatores de proteção). O baixo nível sócio-econômico é uma das variáveis sóciodemográficas mais investigadas em pesquisas sobre fatores de risco (Luthar, 1991). De acordo com Garmezy e Masten (1994), o baixo nível sócio-econômico constitui uma forma de adversidade crônica. Nos anais investigados, essa impressão é confirmada, sendo praticamente a totalidade de estudos referentes a esse público.

Entretanto, é importante salientar que famílias em vulnerabilidade social não se restringem às famílias com baixo nível sócio-econômico. Todos os sistemas familiares enfrentam adversidades que compõem seu processo de desenvolvimento. As atitudes do terapeuta familiar, frente ao sistema que busca terapia, estão fundamentadas na forma como o profissional compreende esses termos. É importante continuar construindo uma rede conceitual precisa que abarque a diversidade do campo da terapia familiar brasileira.

Nos primeiros congressos havia um interesse em caracterizar a família em vulnerabilidade social por meio da descrição do funcionamento interno do sistema (fronteiras, papéis e funções). O mesmo era definido como patológico e as dificuldades para o desenvolvimento saudável de seus membros eram enfatizadas. Nos últimos trabalhos, encontra-se uma alteração substancial na direção dos aspectos positivos dos sistemas familiares. Há um interesse pelos fatores que capacitam as famílias a lidarem e sobreviverem frente a adversidades. $\mathrm{O}$ trabalho do terapeuta familiar passa a ser de captação de recursos e de visibilização da resiliência familiar.

O presente estudo está restrito ao que foi publicado em anais de alguns congressos sobre terapia familiar (resumos). Não houve acesso aos trabalhos completos e nem foram buscadas outras fontes ligadas diretamente à prática da terapia familiar com sistemas em vulnerabilidade social. O interesse de investigação estava voltado aos trabalhos apresentados nos congressos. Acredita-se que todos os conceitos trabalhados neste artigo estão em construção e o espaço de trocas propiciado por encontros entre profissionais estimula discussões que aprimoram sempre mais os mesmos.

\section{Referências}

Aun, J. (2005). Da terapia de família ao atendimento sistêmico à família. Em J. Aun, M. Esteves de Vasconcellos \& S. Coelho (Orgs.), Atendimento sistêmico de famílias e redes sociais (pp. 16-57). Belo Horizonte: Ophicina de Arte \& Prosa.

Bizzo, W. (1994). Encontros e desencontros na relação familiar [Resumo]. Em Associação Brasileira de Terapia Familiar (Org.), Anais do I Congresso Brasileiro de Terapia Familiar (p. 152). São Paulo: ABRATEF.

Cecconello, A. (2003). Resiliência e vulnerabilidade em famílias em situação de risco. Tese de Doutorado, Universidade Federal do Rio Grande do Sul, Porto Alegre.
Cowan, P., Cowan, C., \& Schulz, M. (1996). Thinking about risk and resilience in families. Em E. Hetherington \& E. Blechman (Orgs.), Stress, coping and resiliency in children and families (pp. 1-38). New Jersey: Lawrence Erlbaum.

Dos Santos, J. (2004). A relação pais e filhos no âmbito da escola: uma experiência com famílias de baixa renda [CD-ROM]. Em Associação Brasileira de Terapia Familiar(Org.), Anais do VI Congresso Brasileiro de Terapia Familiar. Florianópolis: ABRATEF.

Felzenswalb, M. (1994). O perfil psico-social da família em contextos de extrema carência [Resumo]. Em Associação Brasileira de Terapia Familiar (Org.), Anais do I Congresso Brasileiro de Terapia Familiar (p. 193). São Paulo: ABRATEF.

Garmezy, N., \& Masten, M. (1994). Chronic adversities. Em M. Rutter, E. Taylor \& L. Hersov (Orgs.), Child and adolescent psychiatry (pp. 191-208). Oxford: Blackwell.

Gonçalves, N. (1996). Ouvindo nossos mestres: integrando teorias e técnicas. Em L. C. Prado (Org.), Famílias e terapeutas construindo caminhos (pp. 37-68). Porto Alegre: Artes Médicas.

Kuperman, S. (2004). Todo adulto diante de uma criança ou de um adolescente é um educador [CD-ROM]. Em Associação Brasileira de Terapia Familiar (Org.), Anais do VI Congresso Brasileiro de Terapia Familiar. Florianópolis: ABRATEF.

Luthar, S. (1991). A study of high-risk adolescents. Child Development, 62, 600-616.

Martinez, D. (1994). Diferentes formas de organização familiar [Resumo]. Em Associação Brasileira de Terapia Familiar (Org.), Anais do I Congresso Brasileiro de Terapia Familiar (p. 34). São Paulo: ABRATEF.

Masten, A., \& Garmezy, N. (1985). Risk, vulnerability and protective factors in developmental psychology. In B. B. Lahey \& A. E. Kazdin (Eds.), Advances in child clinical psychology (pp. 1-52). New York: Plenum Press.

Pesce, R. P., Assis, S. G., Santos, N. C., \& Carvalhaes, R. (2004). Risco e proteção: em busca de um equilíbrio promotor de resiliência. Psicologia: Teoria e Pesquisa, 20, 135-143.

Prado, L. (2001). Terapia familiar breve de famílias com bebês [Resumo]. Em Internacional Family Therapy Association (Org.), Anais do XIII Congresso Internacional de Terapia Familiar (p. 114). Porto Alegre: IFTA.

Rapizo, R. (1998). Terapia sistêmica de família - da instrução à construção. Rio de Janeiro: Instituto NOOS.

Rutter, M. (1987). Psychosocial resilience and protective mechanisms. American Journal of Orthopsychiatric, 57, 316-331.

Rutter, M. (1999). Resilience concepts and findings: Implications for family therapy. Journal of Family Therapy, 21, 119-144.

Sarti, C. (1994). A família pobre [Resumo]. Em Associação Brasileira de Terapia Familiar (Org.), Anais do I Congresso Brasileiro de Terapia Familiar (p. 35). São Paulo: ABRATEF.

Seligman, M. E. P. (2002). Authentic happiness: Using the new positive psychology to realize your potential for lasting fulfillment. New York: Free Press/Simon and Schuster.

Silveira, A. (1998). A terapia familiar e as famílias brasileiras [Resumo]. Em Associação Brasileira de Terapia Familiar (Org.), Anais do III Congresso Brasileiro de Terapia Familiar (p. 17).São Paulo: ABRATEF.

Tejadas, S. (2002). A ética no trabalho com famílias de camadas populares [Resumo]. Em Associação Brasileira de Terapia Familiar (Org.), Anais do V Congresso Brasileiro de Terapia Familiar. Salvador: ABRATEF. Retirado em 25/04/2005, de www.abratef. org.br 
Timoteo, M., \& Falcão, G. (2004). Uma intervenção terapêutica com jovens em situação de risco num bairro de periferia de Salvador [CD-ROM]. Em Associação Brasileira de Terapia Familiar (Org.), Anais do VI Congresso Brasileiro de Terapia Familiar. Florianópolis: ABRATEF.

Vilela, C., Gusmão, C., \& Cavana, M. (2004). Multifamília: quanto o encontro de novas histórias acorda potencialidades e instrumentos terapêuticos [CD-ROM]. Em Associação Brasileira de Terapia Familiar (Org.), Anais do VI Congresso Brasileiro de Terapia Familiar. Florianópolis: ABRATEF.
Walsh, F. (1996). The concept of family resilience: Crisis and challenge. Family Process, 35, 261-281.

Walsh, F. (1998). Strengthening family resilience. New York: Gilford.

Recebido em 06.07.07 Versão final em 17.04.08 Aceito em 29.05.09 